\title{
A NOTE ON AUTOMORPHISM GROUPS OF ALGEBRAIC NUMBER FIELDS
}

\author{
M. FRIED ${ }^{1}$
}

\begin{abstract}
For any finite group $G$ the paper gives an explicit and simple construction of (not necessarily Galois) algebraic extensions of $\mathbf{Q}$ having their full automorphism group equal to $G$.
\end{abstract}

Intrigued by both the result and the last name of one of the authors, we inspected the contents of [EFrK]. In there it is shown that, for any finite group $G$, there is a (not necessarily Galois) extension $L$ of $\mathbf{Q}$ such that the full automorphism group of the extension $L / Q$ is $G$. This is, of course, a weakened form of the celebrated Hilbert-Noether conjecture that every group can be realized as a Galois group over $\mathbf{Q}$. In this note, we make further comment on the nature of the construction of the field $L$; simplify the proof of the existence of $L$; and correct one of the lemmas of [EFrK]. We have been uncompromisingly "generic" in our approach in order to keep technique at a minimum, and also to reveal the many alternatives for the construction of $L$.

First assume that $G$ is contained in $S_{n}$. Let $t_{1}, \ldots, t_{n}$ be algebraically independent indeterminates over $\mathbf{Q}$. It is well known that the splitting field $M_{n}^{(t)}$ of $x^{n}+t_{1} \cdot x^{n-1}+\cdots+t_{n}$ over $\mathbf{Q}\left(t_{1}, \ldots, t_{n}\right)=\mathbf{Q}(\mathbf{t})$ is a regular Galois extension of $Q(t)$ with group equal to $S_{n}$. This is the starting observation of [Hi]: the progenitor of so many notes in the style of this one. Let $M_{G}^{(t)}$ be the fixed field of $G$ in $M_{n}^{(t)}$, and let $\alpha(G, t)$ be a primitive generator of $M_{G}^{(t)}$ over $\mathbf{Q}(t)$.

Let $N$ be any integer greater than 2 and let $z_{1}, \ldots, z_{N}$ be algebraically independent indeterminates over $Q(t)$. Finally, let $\beta(G, t)$ be a zero of $x^{N}+z_{1} \cdot x^{N-1}$ $+\cdots+z_{N-1} \cdot x+\alpha(G, t) \cdot z_{N}$. Now consider the field

$$
L^{(\mathbf{t}, \mathbf{z})}=M_{n}^{(\mathbf{t})}(\beta(G, \mathbf{t}), \mathbf{z})=M_{G}^{(\mathbf{t})}(\beta(G, \mathbf{t}), \mathbf{z}) \cdot M_{n}^{(\mathbf{t})}(\mathbf{z}) .
$$

Then $L^{(t, z)} / M_{G}^{(t)}(\beta(G, t), z)$ is a Galois extension with group

$$
\begin{aligned}
G\left(L^{(\mathbf{t}, \mathbf{z})} / M_{G}^{(\mathbf{t})}(\beta(G, \mathbf{t}), \mathbf{z})\right) & =G\left(M_{n}^{(\mathbf{t})}(\mathbf{z}) / M_{n}^{(t)}(\mathbf{z}) \cap M_{G}^{(\mathbf{t})}(\beta(G, \mathbf{t}), \mathbf{z})\right) \\
& =G\left(M_{n}^{(\mathbf{t})}(\mathbf{z}) / M_{G}^{(t)}(\mathbf{z})\right)=G,
\end{aligned}
$$

our original group.

Suppose that $\sigma$ is any automorphism of $L^{(t, z)} / Q(t, z)$. If $\sigma$ leaves $M_{G}^{(t)}(z)$ fixed, then $\beta(G, t)^{\sigma}$ is another zero of $x^{N}+z_{1} x^{N-1}+\cdots+z_{N-1} x+\alpha(G, t) \cdot z_{N}$. This

Received by the editors August 2, 1979 and, in revised form, October 19, 1979.

1980 Mathematics Subject Classification. Primary 12F05.

Key words and phrases. Galois theory, Hilbert's irreducibility theorem.

${ }^{1}$ This paper was written while the author was a speaker at the 1979 AMS Summer Conference in Group Theory held at Santa Cruz. Supported by NSF Grant MCA-76-07159 and Conference funds. 
implies that $\beta(G, t)^{\sigma}=\beta(G, t)$ since the splitting field of this polynomial over $M_{n}^{(t)}(\mathrm{z})$ is $S_{N}$. Now suppose that $\sigma$ does not fix $M_{G}^{(t)}(\mathrm{z})$. Then $\beta(G, t)$ goes to a root $\beta(G, t)^{\sigma}$ of $x^{N}+z_{1} \cdot x^{N-1}+\cdots+\alpha(G, t)^{\sigma} \cdot z_{N}$. Our next lemma shows that

$$
\beta(G, t)^{\sigma} \notin L^{(t, z)} \quad \text { for each such } \sigma .
$$

With (1) established, $L^{(t, z)}$ is a regular extension of $Q(t, z)$ (its Galois closure over $\mathbf{Q}(\mathbf{t}, \mathbf{z})$ is regular over $\mathbf{Q}(\mathbf{t}, \mathbf{z})$ also) for which the automorphisms of $L^{(t, z)} / \mathbf{Q}(\mathbf{t}, \mathbf{z})$ give the group $G$.

LEMMA. Let $z_{1}, \ldots, z_{N}$ (with $N>1$ ) be algebraically independent indeterminates over a field $M$ of characteristic zero. Let $a_{1}, a_{2} \in M$ be distinct nonzero elements, and let $\beta_{i}$ be a zero of

$$
x^{N}+z_{1} \cdot x^{N-1}+\cdots+z_{N-1} \cdot x+a_{i} \cdot z_{N}, \quad i=1,2 .
$$

Then the fields $M\left(\mathrm{z}, \beta_{1}\right)$ and $M\left(\mathrm{z}, \beta_{2}\right)$ are distinct.

Proof. Suppose that $M\left(\mathrm{z}, \beta_{1}\right)=M\left(\mathrm{z}, \beta_{2}\right)$. Consider the field $L=$ $M\left(z_{1}, \ldots, z_{N-1}\right)$, so that $M\left(\mathrm{z}, \beta_{i}\right)=L\left(z_{N}, \beta_{i}\right), i=1,2$. Let $\bar{L}$ be an algebraic closure of $L$, so that $\bar{L}\left(z_{N}, \beta_{1}\right)=\bar{L}\left(z_{N}, \beta_{2}\right)$ by assumption. The finite branch points of the field extension $\bar{L}\left(z_{N}, \beta_{i}\right) / \bar{L}\left(z_{N}\right)$ with respect to the variable $z_{N}$ consist of the values (in $\bar{L}$ ) of $z_{N}$ for which

$$
N x^{N-1}+(N-1) \cdot z_{1} \cdot x^{N-2}+\cdots+z_{N-1}=0
$$

and equation (2) for " $i$ " have a common solution in $x$. Since $z_{1}, \ldots, z_{N-1}$ are algebraically independent over $M$, these branch points are algebraically independent over $\boldsymbol{M}$. However, these branch points are determined by the field extension, so the two sets of branch points corresponding to $i=1$ and 2 are the same. If $\omega_{1}, \ldots, \omega_{N-1}$ are the zeros of $N x^{N-1}+(N-1) z_{1} \cdot x^{N-2}+\cdots+z_{N-1}=0$, then $-f\left(\omega_{j}\right) / a_{i}, j=1, \ldots, N-1$, runs over the branch points corresponding to $i$, where $f(x)=x^{N}+z_{1} \cdot x^{N-1}+\cdots+z_{N-1} \cdot x$. Thus multiplication by $a_{1} / a_{2}$ maps the branch points corresponding to $i=1$ to the branch points corresponding to $i=2$. This contradicts the algebraic independence of these branch points over $M$.

Finally we prove the main theorem of the paper.

THEOREM. Given any finite group $G$, we can explicitly find an infinite number of field extensions $L / Q$ such that the automorphism group of $L / Q$ is isomorphic to $G$.

Proof. Let $\hat{L}^{(\mathbf{t}, \mathbf{z})} / \mathbf{Q}(\mathbf{t}, \mathbf{z})$ be the Galois closure of the field extension $L^{(t, z)} / \mathbf{Q}(\mathbf{t}, z)$. The automorphism group of $L^{(t, x)} / Q(t, z)$ can be recovered as the quotient $N / G\left(\hat{L}^{(t, z)} / L^{(t, z)}\right)$ where $N$ is the normalizer of $G\left(\hat{L}^{(t, z)} / L^{(t, z)}\right)$ in $G\left(\hat{L}^{(t, z)} / \mathbf{Q}(t, z)\right)$. From Hilbert's irreducibility theorem there are infinitely many specializations $\left(\mathbf{t}_{0}, \mathbf{z}_{0}\right) \in \mathbf{Z}^{n} \times \mathbf{Z}^{N}$ of $(\mathbf{t}, \mathbf{z})$ for which we obtain distinct field extensions $\hat{L}^{\left(\mathbf{t}_{0} z_{0}\right)}$ and $L^{\left(t_{0}, z_{0}\right)}$ over $\mathbf{Q}$ with

$$
G\left(\hat{L}^{\left(\mathrm{t}_{0} \mathrm{z}_{0}\right)} / \mathrm{Q}\right) \simeq G\left(\hat{L}^{(\mathrm{t}, \mathrm{z})} / \mathbf{Q}(\mathbf{t}, \mathrm{z})\right)
$$

and

$$
G\left(\hat{L}^{\left(t_{0}, z_{0}\right)} / L^{\left(t_{0}, z_{0}\right)}\right) \simeq G\left(\hat{L}^{(t, z)} / L^{(t, z)}\right)
$$


Thus we deduce that the automorphism group of $L^{\left(t_{0} z_{0}\right)} / \mathbf{Q}$ is isomorphic to $G$. From the explicit form of Hilbert's irreducibility theorem in [MFr], we may find arithmetic progressions $P^{(t)}$ and $P^{(z)}$ in $\mathbf{Z}^{n}$ and $\mathbf{Z}^{N}$, respectively, such that this holds for $\left(t_{0}, z_{0}\right) \in P^{(t)} \times P^{(z)}$.

The authors of [EFrK] base their proof on the result that there exists a finite undirected graph having neither loops nor isolated points whose automorphism group is $G$ [Fru]. There is a correctable, but significant, error in the proof of their Lemma 2. Let $L$ be a number field, $R$ the ring of integers. If $f_{1}, \ldots, f_{m} \in R[x]$ are monic polynomials that are not pth powers for some prime $p$, then there exists $t \in \mathbf{Z}$ such that $f_{i}(t)$ is not a pth power in $L, i=1, \ldots, t$. The authors conclude that $y^{p}-f_{i}(x)=0$ is not a genus zero curve, and they apply Siegel's theorem to conclude that there are only finitely many integral points. First of all, such a use of Siegel's theorem would make their field construction completely ineffective (which it should not be), and secondly (for a trivial counterexample) take $m=1, p=2$, $f_{1}(x)=x^{3}$ to get a genus zero curve. However, this can be corrected by using Hilbert's irreducibility theorem as in the proof of the theorem above. Let $g_{i}^{()}(x, y)$, $j=1, \ldots, m(i)$, run over the irreducible factors of $y^{p}-f_{i}(x)$. By hypothesis, $g_{i}^{(j)}(x, y)$ is of degree greater than 1 in $y$. By Hilbert's theorem there exists $t \in \mathbf{Z}$ such that $g_{i}^{(j)}(t, y)$ remains irreducible over $\mathbf{Q}$ for $j=1, \ldots, m(i) ; i=1, \ldots, t$.

\section{REFERENCES}

[EFrK] E. Fried and J. Kollár, Automorphism groups of algebraic number fields, Math. Z. 163 (1978), 121-123.

[MFr] M. Fried, On Hilbert's irreducibility theorem, J. Number Theory 6 (1974), 211-232.

[Fru] R. Frucht, Herstellung von Graphen mit Vorgegebener abstrakter Gruppe, Compositio Math. 6 (1938), 239-250.

[Hi] D. Hilbert, Über die Irrechuibilität ganzer rationaler Functionen, mit ganzzahligen Koeffizienten, Crelles J. Math. 110 (1892), 104-129.

Department of Mathematics, University of California, IrVine, California 92717 